\title{
Research on an Improved Medical Image Enhancement Algorithm Based on P-M Model
}

\author{
Luo Aijing ${ }^{1}$ and Yin Jin ${ }^{2, *}$
}

\begin{abstract}
${ }^{I}$ Key Laboratory of Medical Information Research (Central South University), College of Hunan Province; The Third Xiangya Hospital of Central South University; Postal code 410013, Changsha, China; ${ }^{2}$ School of Public Health, Central South University; Xidian University, Postal code 710071, Xi'an, China
\end{abstract}

\begin{abstract}
Image enhancement can improve the detail of the image to achieve the purpose of the identification of the image. At present, the image enhancement is widely used in medical images, which can help doctor's diagnosis. IEABPM (Image Enhancement Algorithm Based on P-M Model) is one of the most common image enhancement algorithms. However, it may cause the loss of the texture details and other features. To solve the problems, this paper proposes an IIEABPM (Improved Image Enhancement Algorithm Based on P-M Model). The simulation demonstrates that IIEABPM can effectively solve the problems of IEABPM, and improve image clarity, image contrast, and image brightness.
\end{abstract}

Keywords: Bending, distortion, image enhancement, Matlab, P-M model, tilt.

\section{INTRODUCTION}

In the field of image processing, image enhancement is a very important research direction, which has been widely used in military, remote sense, public safety, biomedicine, etc [1]. In the field of medicine, the image is usually gathered by CT machine, ultrasonic apparatus, and so on. The collected images may be blurry, which will affect diagnoses of the illness. To improve the quality of the collected images, image enhancement is used [2].

From the aspect of the realization, the image enhancement algorithm can be divided into five categories: image enhancement algorithm based on traditional theory, image enhancement algorithm based on multiscale analysis [3], image enhancement algorithm based on fuzzy theory [4], image enhancement algorithm based on humanoid vision [5] and image enhancement algorithm based on mathematic morphology [6]. Among them, the image enhancement algorithm based on mathematic morphology consists of closing operation, erosion operation, dilation operation and open operation. IEABPM (Image Enhancement Algorithm Based on P-M Model) is one of the most widely used [7, 8]. IEABPM can effectively remove the noise of images, however, for the area which has rich textures, it may cause the loss of the texture details and other features. To solve the problem, IIEABPM (Improved Image Enhancement Algorithm Based on P-M Model) is proposed. And Simulation demonstrates that IIEABPM can improve image clarity, image contrast, entropy and image brightness.

\section{IIEABPM}

In IIEABPM, it firstly uses the normalization method to translate P-M model into the posed problem. Secondly, a moderator is added to control the process of optimization.

*Address correspondence to this author at the School of Public Health, Central South University; Xidian University, Postal code 710071, Xi'an, China; Tel: 86-29-88204338; E-mail: jyin@xidian.edu.cn
Thirdly, according to different region status of the image, IIEABPM chooses the spread function. To meet the performance requirements, the spread function is corrected through four steps. The first step is increasing the gradient threshold. The second step is modifying spread function. The third step is adding gradient fidelity term. The last step is adding strength coefficient. Fig. (1) gives the flowchart of IIEABPM.

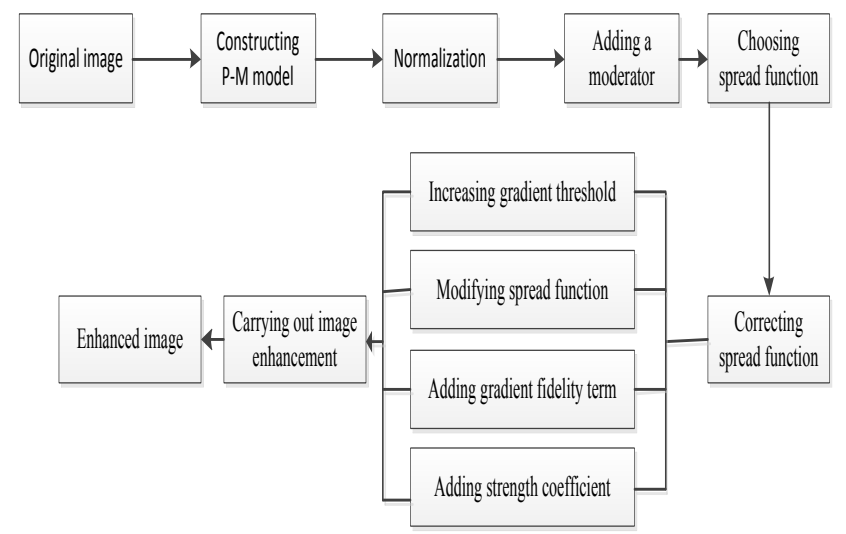

Fig. (1). Flow chart of IIEABPM.

According to Fig. (1), when the normalization is used, the Gaussian kernel is adopted to smooth the images.

$u_{t}=\operatorname{div}\left(c\left(\left|\nabla u_{\sigma}\right|\right) \nabla u\right)$

Where $u_{\sigma}(x, y, t)=G_{\sigma} * U(x, y, t)$, and $G_{\sigma}$ is a Gaussian function, whose mean value and variance are respectively 0 and $\sigma^{2}$.

After normalization, P-M model is translated into a posed problem, and there is only one continuous solution, which depends on the initial value $u_{0}(x, y)$. And then a moderator is added to control the process of optimization, and 
$u(x, 0)=u_{0}(x) \rightarrow u(x, y, 0)=u_{0}(x, y)$. Therefore, the mathematical model of image enhancement can be written as follows:

$$
\begin{aligned}
& \frac{\partial u_{t}(x, y)}{\partial t}=\operatorname{div}\left(g\left(\nabla u_{t}\right) \nabla u_{t}\right) \\
& u(x, y, 0)=u_{0}(x, y)
\end{aligned}
$$

Where $u$ is the image at time $t$, div means divergence operator, $\nabla u_{t}$ is the gradient of the image, $g\left(\nabla u_{t}\right)$ is the spread function, whose value means the strength of spread. In some area of the image, the value of $\left|\nabla u_{t}\right|$ decides whether or not the image is smooth. That is to say, when the value of $\left|\nabla u_{t}\right|$ is smaller, the image in the region is smoother.

In order to keep the anisotropic diffusion of spread function, $\nabla u_{t}$ must make spread function satisfy the following two conditions:

(1) The spread of the noise is within the relatively smooth featured area;

(2) The spread does not take place between two adjacent areas to preserve the edge details.

When removing the noise of the image, spread function can be chosen from two kinds of expressions:

$$
\begin{aligned}
& g\left(\left|\nabla u_{t}\right|\right)=\frac{1}{1+\left(\frac{\left|\nabla u_{t}\right|}{k}\right)^{2}} \\
& g\left(\left|\nabla u_{t}\right|\right)=e^{-\left(\frac{\left|\nabla u_{t}\right|}{k}\right)^{2}}
\end{aligned}
$$

\subsection{The First Step}

Although the above spread model has good effects on the image denoise, its effect is not ideal when the noise is high. To solve the problem, Eq. (3) and Eq. (4) can be modified:

$g\left(\left|\nabla u_{t}\right|\right)=\frac{k_{3}}{1+\left(\frac{\left|\nabla u_{t}\right|}{k_{1}+k_{2}}\right)^{2}}$

$g\left(\left|\nabla u_{t}\right|\right)=e^{-\left(\frac{\left|\nabla u_{t}\right| k_{3}}{k_{1}+k_{2}}\right)^{2}}$

Where $k_{1}, k_{2}, k_{3}$ are the gradient thresholds, and $k_{1} \approx k_{3}$, $k_{1}, k_{3}>k_{2}$. Fig. (2) shows the relationships between spread function and gradient.

From Fig. (2), we can see that the gradient and spread function has an inverse relationship. And compared with Eq. (3) and Eq. (4), Eq. (5) and Eq. (6) can better reserve the edge and texture.

\subsection{The Second Step}

In order to more accurately control the smoothness, $g\left(\left|\nabla u_{t}\right|\right)$ is replaced by $g\left(\left|\nabla G_{\sigma} * u\right|\right)$, where the expression of $G_{\sigma}$ is:
$G_{\sigma}=\frac{c}{\sqrt{\sigma}} \exp \left(-\frac{x^{2}+y^{2}}{4 \sigma}\right)$

Therefore, divergence operator can be written as:

$$
\frac{\partial u}{\partial t}=\operatorname{div}\left(g\left(\left|\nabla G_{\sigma} * u\right|\right)|\nabla u|\right)
$$

Where,

$$
g(x, y, t)=\exp \left(-\left(\frac{\nabla G_{\sigma} * u}{k}\right)^{2}\right) .
$$

When calculating $\nabla G_{\sigma} * u_{0}$, the similarity functional of two signals is:

$$
E(u)=\int_{\Omega} a\left(u-u_{0}\right)^{2}+\beta\left(\left\|\nabla u-\nabla\left(\nabla G_{\sigma} * u_{0}\right)\right\|\right)^{2} d x d y
$$

Where $\alpha$ and $\beta$ are weight coefficients,

$\left(\left\|\nabla u-\nabla\left(\nabla G_{\sigma} * u_{0}\right)\right\|\right)^{2}$

is the gradient fidelity term, which tries to make changes in the gradient which are consistent with $\nabla\left(G_{\sigma} * u_{0}\right)$.

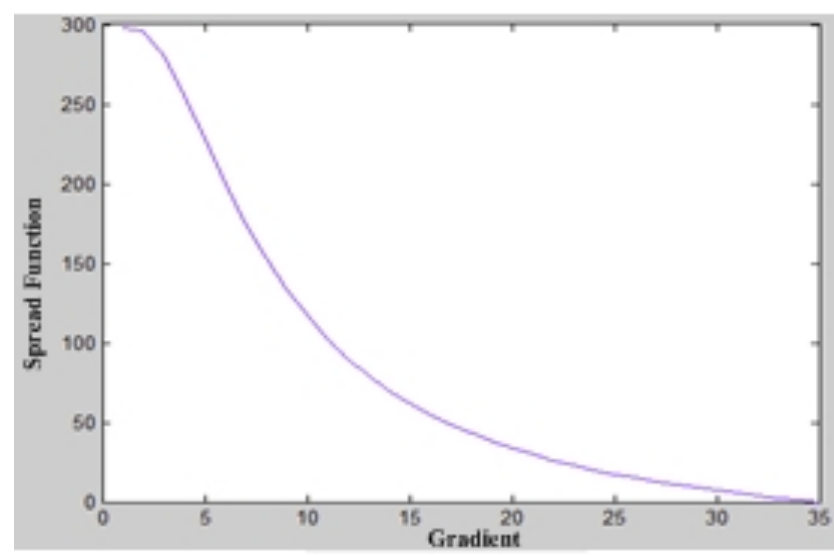

Fig. (2). Gradient vs. Spread Function.

\subsection{The Third Step}

In Eq. (9), gradient fidelity term is added to increase the smoothness of the image. However, whether it will impede the optimal solution has not been proved. If $E(u)$ is the convex function, the optimal solution uniquely exists.

Theorem 1 if $\lambda_{1}, \lambda_{2}>0, \lambda_{1}+\lambda_{2}=1$, $E\left(\lambda_{1} u_{1}+\lambda_{2} u_{2}\right) \leq \lambda_{1} E\left(u_{1}\right)+\lambda_{2} E\left(u_{2}\right)$.

Proof:

$$
\begin{aligned}
& \left(\lambda_{1} u_{1}+\lambda_{2} u_{2}-u_{0}\right)^{2}=\left[\lambda_{1}\left(u_{1}-u_{0}\right)+\lambda_{2}\left(u_{2}-u_{0}\right)\right]^{2} \\
& =\lambda^{2}{ }_{1}\left(u_{1}-u_{0}\right)^{2}+\lambda^{2}{ }_{2}\left(u_{2}-u_{0}\right)^{2}+2 \lambda_{2} \lambda_{1}\left(u_{1}-u_{0}\right)\left(u_{2}-u_{0}\right) \\
& =\lambda^{2}{ }_{1}\left(u_{1}-u_{0}\right)^{2}+\lambda^{2}{ }_{2}\left(u_{2}-u_{0}\right)^{2}-\lambda_{2} \lambda_{1}\left[\left(u_{1}-u_{0}\right)\left(u_{2}-u_{0}\right)\right]^{2} \\
& \leq \lambda_{1}^{2}\left(u_{1}-u_{0}\right)^{2}+\lambda_{2}^{2}\left(u_{2}-u_{0}\right)^{2}
\end{aligned}
$$


Then,

$$
\begin{aligned}
& \left(\left\|\nabla\left(\lambda_{1} u_{1}+\lambda_{2} u_{2}\right)-\nabla\left(G_{\sigma} * u_{0}\right)\right\|\right)^{2}= \\
& \left(\| \lambda_{1} \nabla u_{1}-\lambda_{1} \nabla\left(G_{\sigma} * u_{0}\right)+\lambda_{2} \nabla u_{2}\left|-\lambda_{2} \nabla\left(G_{\sigma} * u_{0}\right)\right|\right) \\
& =\lambda_{1}^{2}\left(\left\|\nabla u_{1}-\nabla\left(G_{\sigma} * u_{0}\right)\right\|\right)^{2}+\lambda_{1}^{2}\left(\left\|\nabla u_{2}-\nabla\left(G_{\sigma} * u_{0}\right)\right\|\right)^{2} \\
& +2 \lambda_{1} \lambda_{2}\left(\nabla u_{1}-\nabla\left(G_{\sigma} * u_{0}\right)\right)\left(\nabla u_{2}-\nabla\left(G_{\sigma} * u_{0}\right)\right) \\
& \leq \lambda_{1}^{2}\left(\left\|\nabla u_{1}-\nabla\left(G_{\sigma} * u_{0}\right)\right\|\right)^{2}+\lambda_{1}^{2}\left(\left\|\nabla u_{2}-\nabla\left(G_{\sigma} * u_{0}\right)\right\|\right)^{2}
\end{aligned}
$$

Therefore,

$$
E\left(\lambda_{1} u_{1}+\lambda_{2} u_{2}\right) \leq \lambda_{1} E\left(u_{1}\right)+\lambda_{2} E\left(u_{2}\right) .
$$

An image can be regarded as the surface in twodimensional space. The aim of using the gradient fidelity term is to keep the constraint of the continuity of the image topology. In the iterative computation, the gradient fidelity term maintains consistency of the original image and enhanced image, which can eliminate the loss of the image texture details and other features. Based on the above analysis, Eq. 8 can be modified as:

$$
\frac{\partial u}{\partial t}=\operatorname{div}\left(g\left(\left|\nabla G_{\sigma} * u\right|\right)|\nabla u|\right)-a\left(\nabla u-\nabla\left(\nabla G_{\sigma} * u\right)\right)
$$

Where $\alpha$ is the weight coefficient, $\alpha>0$.

\subsection{The Fourth Step}

To increase detailed information, the strength coefficient is added to the spread model. The model is:

$$
\frac{\partial u_{t}(x, y)}{\partial t}=\operatorname{div}\left(g\left(\left|\nabla G_{\sigma} * u\right|\right) \nabla u_{t}\right)+e^{w} \times u_{t}
$$

Where $w_{\text {is }}$ the strength coefficient, div means divergence operator.

\section{SIMULATION RESULTS}

\subsection{Enhancement Effect Analysis}

To verify the effectiveness of IIEABPM, enhancement effect tests were conducted, as shown in Fig. (3).

In Fig. (3), the original images are (a1), (b1) and (c1), which are, respectively, hand bone image, angiocarpy image and rib image. (a2), (b2) and (c2) are enhanced images, accordingly. In the test, $k_{1}=32, k_{2}=7, k_{3}=32, \Delta t=0.0001$, $\mathrm{n}=25$. From Fig. (3), we can see that enhanced images become clearer, and texture details have also been retained.

\subsection{Performance Analysis}

To further analyze the performance of IIEABPM, three tests have been designed.

In the first test, Fig. 3(b1) was chosen as the subject and the performance of IEABPM and IIEABPM was compared in terms of the clarity, contrast, brightness and entropy. Fig. (4) gives the enhanced images, and Table 1 gives features of the enhanced images.

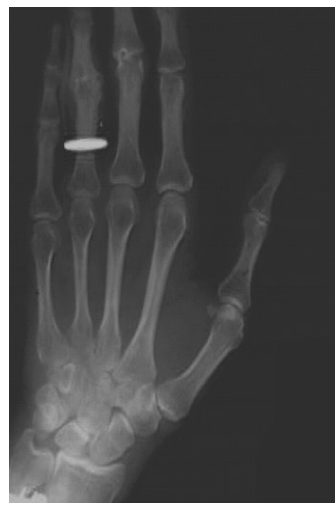

(a1)

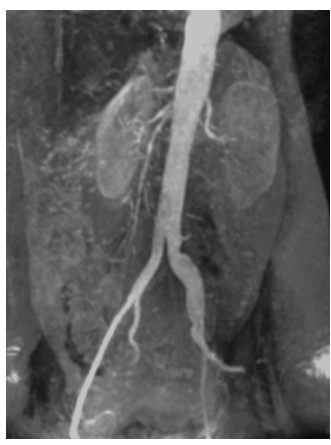

(b1)

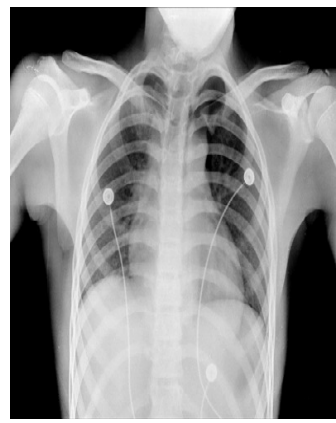

(c1)

Fig. (3). Enhancement effect.

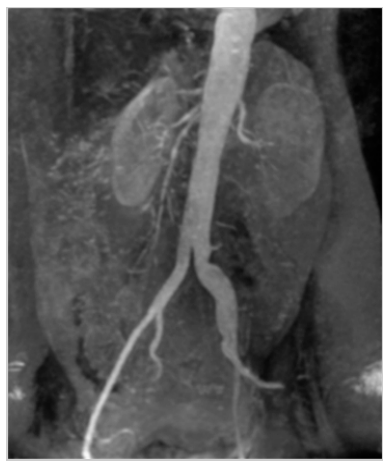

(a) Enhanced Image by IEABPM

Fig. (4). Enhanced images.

From Fig. (4) and Table 1, we can see that the performance of IIEABPM is better than that of IEABPM.

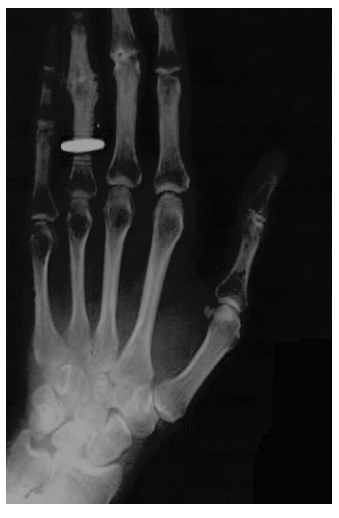

(a2)

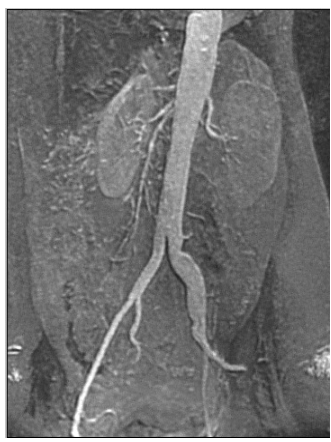

(b2)

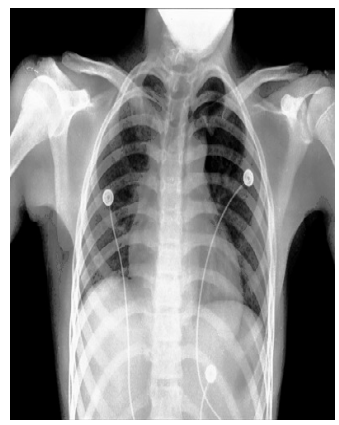

(c2)

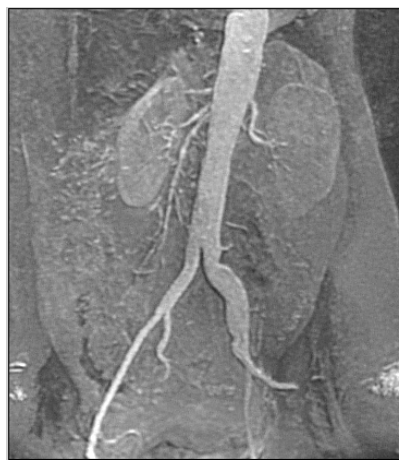

(b) Enhanced Image by IIEABPM 
Table 1. Features of images.

\begin{tabular}{|c|c|c|c|c|}
\hline & Brightness & Contrast & Entropy & Clarity \\
\hline \hline Original image & 57.15 & 311.36 & 6.29 & 38.21 \\
\hline IEABPM & 77.42 & 441.67 & 10.18 & 59.83 \\
\hline IIEABPM & 79.26 & 487.82 & 14.01 & 73.85 \\
\hline
\end{tabular}

In the second test, Fig. (4a) with noise was chosen as the subject. The performance of image enhancement by the median filter, image enhancement by Gaussian filter, image enhancement by wavelet filter, IIEABPM and IEABPM were compared in terms of the enhancement effect, as shown in Fig. (5).

In Fig. (5), we can see that the performances of IEABPM and IIEABPM are better than the performances of the other. In Fig. (5e), many details have been removed. However, in Fig. (5f), not only the image noise is restrained, but details were also reserved.

In the third test, Fig. (3a) was chosen as the subject. The performance of image enhancement by the median filter, image enhancement by Gaussian filter, image enhancement by wavelet filter, IIEABPM and IEABPM were compared in terms of the enhancement effect, as shown in Fig. (6).

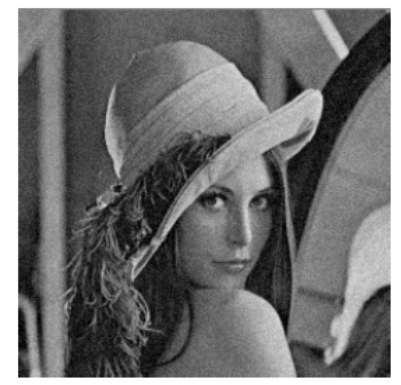

(a) Lena

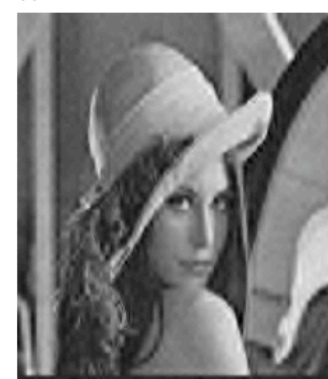

(d) Enhanced Image by Wavelet Filter

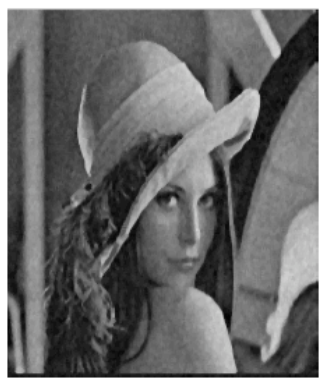

(b) Enhanced Image by Median Filter

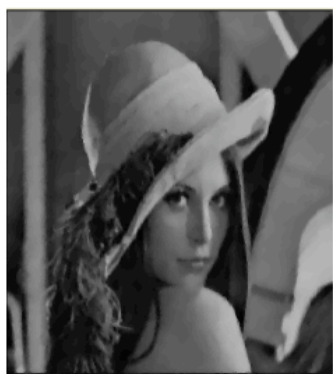

(e) Enhanced Image by IEABPM

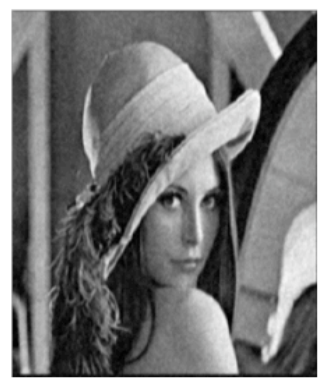

(c) Enhanced Image by Gaussian Filter

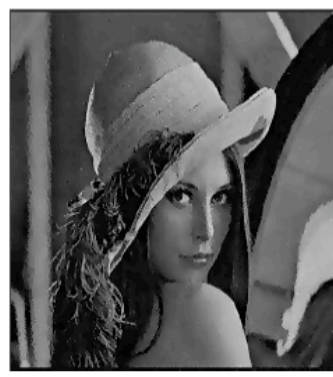

(f) Enhanced Image by IIEABPM

Fig. (5). Compared results for Lena.

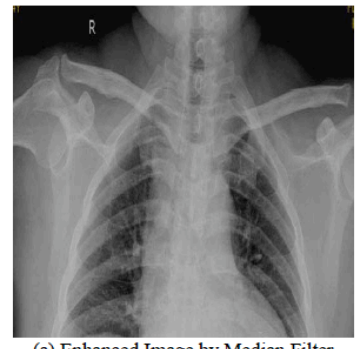

(a) Enhanced Image by Median Filter

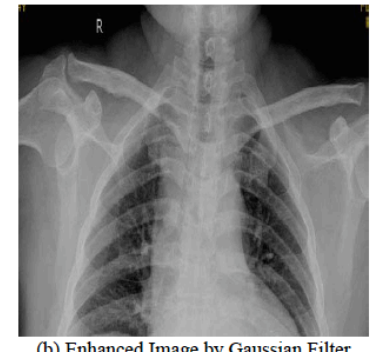

(b) Enhanced Image by Gaussian Filter

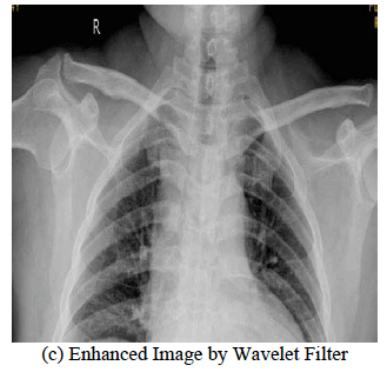

(c) Enhanced Image by Wavelet Filter

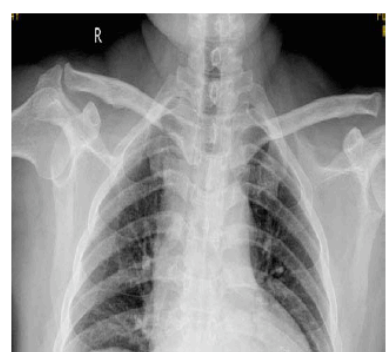

(d) Enhanced Image by IEABPM

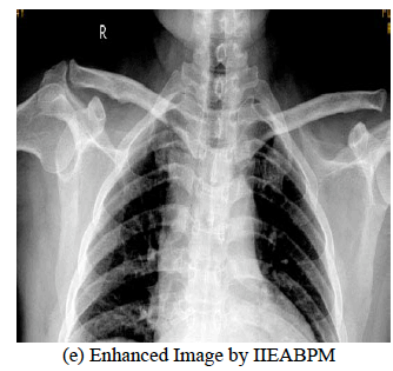

Fig. (6). Compared results for rib image. 
From Fig. (6), we can see that the performance of IIEABPM is the best.

\section{CONCLUSION}

To solve the problems of IEABPM, the paper proposes IIEABPM, and simulation demonstrates that IIEABPM can effectively improve image clarity, image contrast, and image brightness.

\section{CONFLICT OF INTEREST}

The authors confirm that this article content has no conflict of interest.

\section{ACKNOWLEDGEMENTS}

This work was supported by the Youth Foundation of the Education Department of Hebei Province (QN2014182), Science and Technology Department of Hebei Province (112135119), Youth Foundation of Natural Science of Hebei North University (Q2014008), Youth Foundation of Natural Science of Hebei North University (Q2014002).

\section{REFERENCES}

[1] M. P. Ekstrom, Digital Image Processing Techniques, Academic Press, 2012.

[2] H. Wang, Y. Pan, and K. Chen, "Enhancement of low-dose lung CT image based on stochastic resonance of FHN neurons," Hangtian Yixue yu Yixue Gongcheng, vol. 25, pp. 121-125, 2014.

[3] S. S. Bedi, and R. Khandelwal, "Various image enhancement techniques-a critical review," International Journal of Advanced Research in Computer and Communication Engineering, vol. 2, pp. 251-257, 2013.

[4] D. K. Patel, and S. A. More, "An enhanced approach for edge image enhancement using fuzzy set theory and cellular learning automata (CLA)," World Journal of Science and Technology, vol. 2, pp. 158-162, 2012.

[5] I. Busheri, and A. Herman, "Digital image enhancement improves diagnosis of non displaced proximal femur fractures," Clinical Orthopedics and Related Research, vol. 32, pp. 432-453, 2008.

[6] Y. Kimori, "Mathematical morphology-based approach to the enhancement of morphological features in medical images," Journal of Clinical Bioinformatics, vol. 1, pp. 1-10, 2011.

[7] Y. Cai, and Y. Huang, "Image smoothing process model and improving based on P\&M model," Computer Simulation, vol. 28, pp. 256-273, 2011

[8] Y. Mu, and Y. Yu, "Reasearch on image filtering algorithm," Computer Engineering, vol. 2, pp.32-37, 2014. 